Research Paper

\title{
Real-World Data on Prognostic Factors for Overall Survival in EGFR-Mutant Non-Small-Cell Lung Cancer Patients with Brain Metastases
}

\author{
Xiaoqing $\mathrm{Yu}^{1}$, Yun Fan ${ }^{1,2}{ }^{\bowtie}$ \\ 1. Department of Thoracic Medical Oncology, Zhejiang Caner Hospital, Hangzhou, 310022, Zhejiang, People's Republic of China \\ 2. Key laboratory Diagnosis and Treatment Technology on Thoracic Oncology (esophagus, lung), Zhejiang Caner Hospital, 310022, Zhejiang, People's \\ Republic of China \\ $\triangle$ Corresponding author: Dr. Yun Fan, Department of Thoracic Medical Oncology; Key laboratory Diagnosis and Treatment Technology on Thoracic Oncology \\ (esophagus, lung), Zhejiang Caner Hospital, No 1, East Banshan Road, Gongshu District, Hangzhou, 310022, Zhejiang, People's Republic of China. Fax +86571 \\ 8812 2589. Email: fanyun_zjcc@163.com \\ (0) Ivyspring International Publisher. This is an open access article distributed under the terms of the Creative Commons Attribution (CC BY-NC) license \\ (https://creativecommons.org/licenses/by-nc/4.0/). See http://ivyspring.com/terms for full terms and conditions.
}

Received: 2018.09.30; Accepted: 2019.04.28; Published: 2019.06.09

\begin{abstract}
Background: With the wide application of epidermal growth factor receptor-tyrosine kinase inhibitors (EGFR-TKIs), the survival of EGFR-mutant non-small-cell lung cancer (NSCLC) patients with brain metastasis (BM) has been significantly improved. However, prognosis analysis for patients with EGFR mutation and BM is still lacking, and the prognostic factors remain to be determined.

Materials and methods: $A$ total of 746 NSCLC patients with BM were identified between January 2013 and December 2016 at our institution. Overall, 261 patients harboring EGFR mutation and meeting the inclusion criteria for the study were enrolled. Exclusion criteria included KPS $<50$, diagnosed with BM during treatment with EGFR-TKIs, or insufficient follow-up. Overall survival (OS) was measured from the date of brain metastases. Independent prognostic factors were confirmed using a Cox regression model.

Results: The median follow-up time for these patients was 32.7 months ( $95 \% \mathrm{Cl}, 23.5-41.9)$. The median OS after development of brain metastases was 23.0 months $(95 \% \mathrm{Cl}, 20.01-25.99)$. By univariate analysis, significantly shorter OS was noted in patients older than 65 years $(p=0.025)$, KPS $<70(p=0.003)$, presence of extracranial metastases $(E C M)(p=0.00)$, without intracranial local treatment $(p=0.000)$, and without chemotherapy $(p=0.001)$. There was no difference in OS with respect to EGFR mutation type and number of $B M(p=0.343, p=0.729$, respectively). The Cox proportional hazards regression model revealed that performance status $(K P S<70, p=0.010)$, ECM $(p=0.001)$, receiving intracranial local treatment $(p=0.005)$ and chemotherapy $(p=0.005)$ were independent prognostic factors for OS, while age was not $(p=0.087)$. Patients with higher diagnosis-specific graded prognostic assessment (DS-GPA) and Lung-molGPA scores corresponded to better prognosis $(p=0.000)$.

Conclusion: This retrospective analysis demonstrated that performance status (KPS $\geq 70$ ), absence of ECM metastases, administration of local treatment and chemotherapy were associated with superior OS in patients with EGFR-mutant NSCLC who developed BM. The DS-GPA and Lung-molGPA indexes still applied to NSCLC patients with mutant genotypes and BM.
\end{abstract}

Key words: Non-Small-Cell Lung Cancer, Brain Metastases, Prognostic Factors, Epidermal Growth Factor Receptor Tyrosine Kinase Inhibitors

\section{Introduction}

Brain metastasis (BM) has become a leading cause of death from non-small-cell lung cancer
(NSCLC), with an incidence of approximately $25-40 \%$ $[1,2]$. As systemic therapies improve, NSCLC patients 
live longer and are thus at an increased risk for brain metastases. For patients harboring epidermal growth factor receptor (EGFR) mutations, the incidence of BM is up to $50 \%$, which seriously affects patient prognosis [3]. The reported frequency of somatic EGFR mutations varies from $30 \%$ to $50 \%$ among East Asians to approximately $10 \%$ among Caucasians [4]. Currently, EGFR-tyrosine kinase inhibitor (TKI) is the standard first-line treatment for patients with advanced, recurrent, or metastatic NSCLC harboring an EGFR mutation [5]. Preclinical data have demonstrated that gefitinib, icotinib and erlotinib can penetrate the blood-brain barrier (BBB) at concentrations adequate for initiating antitumor activity [6, 7]. The reported cerebrospinal fluid (CSF)/plasma concentrations of the first-line EGFR TKIs are approximately 0.6-2.5\%[8-10]. Many retrospective and prospective studies have confirmed that EGFR TKIs have significant intracranial activity $[9,11]$. A large phase 3 , randomized controlled trial (BRAIN) reported by $\mathrm{Wu}$ et al. examined the efficacy of icotinib and whole-brain irradiation (WBI) in primary diagnosed NSCLC patients with EGFR mutations and at least three metastatic brain lesions [12]. The results showed icotinib has superior iPFS, PFS and ORR over WBI in EGFR-mutant advanced NSCLC with BM and has a well-tolerated safety profile. Therefore, the therapeutic status of EGFR TKIs in EGFR-mutant patients with BM has been confirmed. However, historically, brain metastases have been treated with surgical resection, stereotactic radiosurgery (SRS), or whole-brain radiotherapy (WBRT), either alone or in combination. Recently, a retrospective study reported in Journal of Clinical Oncology by Magnuson and his colleagues demonstrated that the use of upfront EGFR-TKI, and deferral of radiotherapy, is associated with inferior overall survival (OS) in patients with EGFR-mutant NSCLC who develop brain metastases [5]. Therefore, despite the availability of various treatment options in this group of patients with BMs, including EGFR-TKIs, WBRT, and SRS, the optimal treatment combination or sequence has been unclear. BM is a complex and genetically heterogeneous population. Extensive efforts have focused on predicting outcomes for BM patients. The prognostic indexes have been widely applied in clinical practice, such as the Graded Prognostic Assessment (GPA) and the Disease-Specific GPA (DS-GPA). However, these indexes were mostly based on BM patients with unknown genetic status. Recently, an update of the DS-GPA using molecular markers (Lung-molGPA) was proposed by Paul W. Sperduto, incorporating gene alteration data into the DS-GPA. However, the prognostic value of the Lung-molGPA models for patients with EGFR mutation remains undetermined. The recent revolution in the treatment of patients with predictive biomarkers brought about the significant improvement of survival outcomes; thus, we sought to describe outcomes for patients with NSCLC and EGFR mutations and to identify prognostic factors for survival in a real-world population of EGFR-mutant NSCLC patients with BM that can be used to appropriately tailor treatment strategies. We also wanted to evaluate the therapeutic value and the prognostic effect of intracranial local therapy, chemotherapy and comprehensive treatments on patient survival. Meanwhile, we evaluate the prognostic value of the DS-GPA and Lung-molGPA indexes.

\section{Materials and methods}

\section{Patients and Populations}

In this retrospective study, all patients aged 18 years or older with a diagnosis of NSCLC and with magnetic resonance imaging (MRI) confirmed BM diagnoses at our institution from January 2013 to December 2016 were identified. Other eligibility criteria included the following: harboring EGFR mutations and treatment with EGFR TKIs. Exclusion criteria included the following: KPS $<50$, diagnosed with BM during treatment with EGFR TKIs, and loss to follow-up. Baseline clinical characteristics were recorded by retrospective chart review, including age at diagnosis of BM, sex, smoking history, KPS, tumor histology, number of BM, ECM, whether the patient was symptomatic from $\mathrm{BM}$ and synchronous $\mathrm{BM}$, type of EGFR mutation, intracranial local therapy delivered, and chemotherapy that was delivered. Patient follow-up by telephone was done until April 2018. Treatment response was evaluated, and survival data were collected and analyzed. This investigation was approved by the Zhejiang Center Hospital Ethics Committee.

\section{Assessment}

MRI scans of the brain, along with positron emission tomography-computed tomography (CT) scans of the chest, were reviewed every 2 months. Tumor response to treatment was evaluated based on Response Evaluation Criteria in Solid Tumors version 1.1[13]. The primary outcome of this study was OS, defined as the duration between the diagnosis of BM and the time of death (all causes).

\section{Statistical Analysis}

Patient OS was assessed using the Kaplan-Meier method. The log-rank test was used for comparison of survival curves of different characteristics. Prognostic factors for OS were analyzed by univariate and 
multivariate analyses. Variables with $p<0.1$ were added to the final multivariate Cox regression model. The hazard ratio (HR) and 95\% confidence interval (CI) were calculated for all variables in the regression model. Statistical significance was set at $p<0.05$. All statistical analyses were performed using SPSS software, version 20.0 (SPSS Inc., Chicago, IL, USA).

\section{Results}

\section{Patient characteristics}

From January 1, 2013, to December 31, 2016, a total of 746 patients were diagnosed with NSCLC with BM at Zhejiang Cancer Hospital. Of these patients, 269 EGFR-mutant patients had been treated with EGFR TKIs after the diagnosis of BM. After excluding those who met the exclusion criteria, 261 patients were enrolled in the study (Fig. 1). The median follow-up time for these patients was 32.7 months (95\% CI 23.5-41.9). The median age was 54 years (range 30-79 years). The patients were predominantly younger than 65 years old $(\leq 65$ years old, $85.8 \%$ ), female (59.4\%), with a KPS score $\geq 70$ $(71.6 \%)$, histology of adenocarcinoma $(92.0 \%)$, lower number of BM (number of BM lesions $\leq 3,67 \%$ ), without ECM (55.2\%), without symptomatic BM (56.3\%), with synchronous BM $(72.4 \%)$, receiving local therapy (74.7\%) and chemotherapy (56.3\%). All patients were stratified according to the criteria of DS-GPA and Lung-molGPA. Overall, 42 patients $(16.1 \%)$ had a DS-GPA score of 0-0.5, 102 patients $(41.0 \%)$ were $1-1.5,86$ patients $(33.0 \%)$ were $2-2.5$, and 26 patients $(10.0 \%)$ were more than 3 . For the Lung-molGPA index [3], 7 patients $(2.7 \%)$ were $0-1,71$ $(27.2 \%)$ were $1.5-2,125(47.9 \%)$ were $2.5-3$, and 58 $(22.2 \%)$ were above 3.5. These EGFR-mutant cases included $138(52.9 \%)$ exon 19 deletion, 90 (34.5\%) L858R mutation, and 33 (12.6\%) other EGFR mutations (including the presence of T790M mutation during treatment and other uncommon mutations). Due to medical insurance limitations, icotinib was not covered by Zhejiang Medicare until June 2016, whereas gefitinib and erlotinib were not covered until September 2017. Therefore, $38.8 \%$ patients received chemotherapy as first-line treatment in this study. Up to $69.3 \%$ patients had received icotinib in our study. Platinum doublet-chemotherapy was the most common chemotherapy regimen. The patient characteristics at baseline are detailed in Table 1.

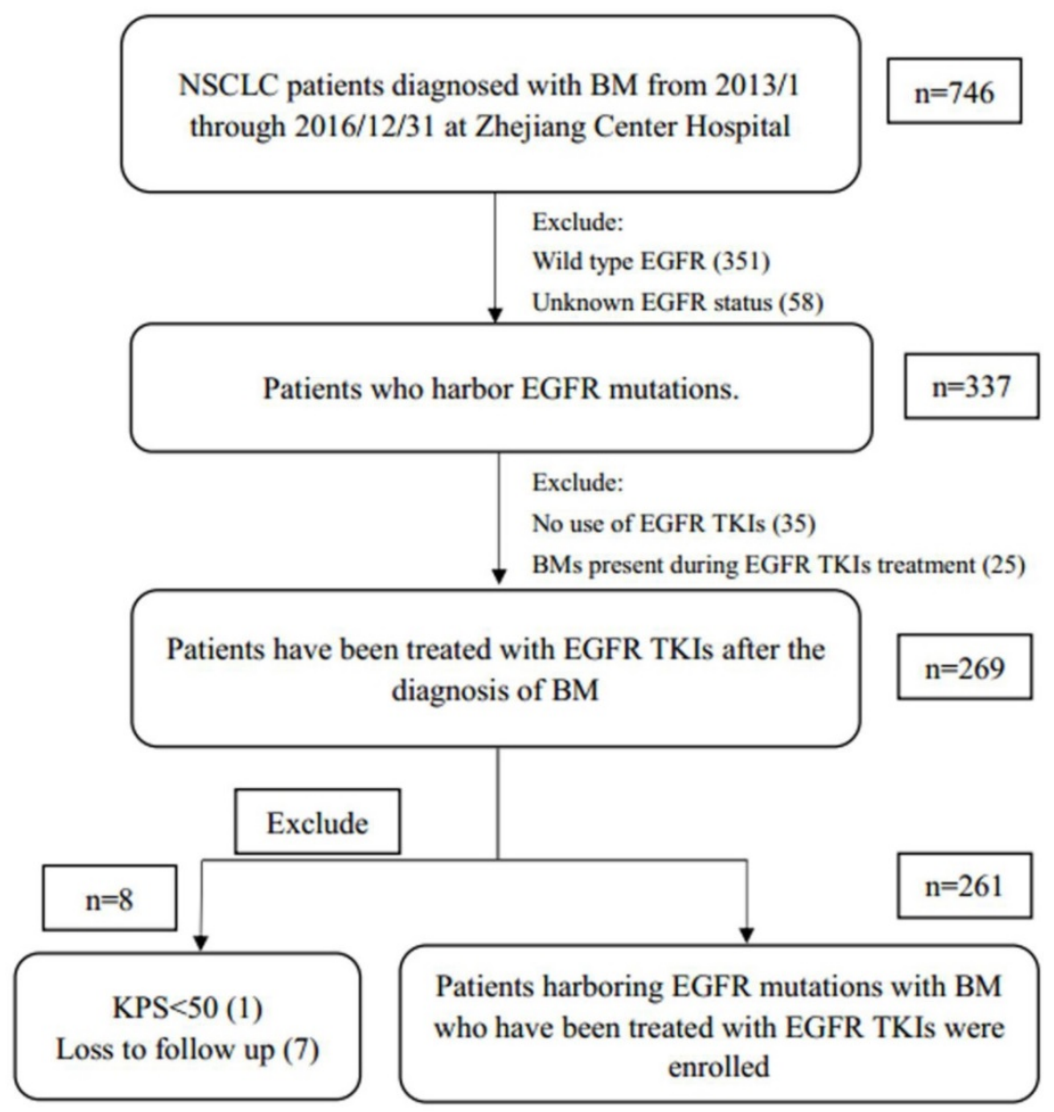

Figure 1. Patient selection and exclusion criteria. Abbreviations: NSCLC, non-small cell lung cancer; BM, brain metastases; EGFR, epidermal growth factor receptor; TKI, tyrosine kinase inhibitor; KPS, Karnofsky performance score. 
Table 1. Baseline characteristics

\begin{tabular}{|c|c|c|}
\hline Characteristic & $\mathrm{n}$ & $(\%)$ \\
\hline \multicolumn{3}{|l|}{ Age (30-79 years) } \\
\hline$\leq 65$ years old & 224 & $(85.8 \%)$ \\
\hline$>65$ years old & 37 & $(14.2 \%)$ \\
\hline \multicolumn{3}{|l|}{ Gender } \\
\hline Male & 106 & $(40.6 \%)$ \\
\hline Female & 155 & $(59.4 \%)$ \\
\hline \multicolumn{3}{|l|}{ Smoking history } \\
\hline Smoker & 72 & $(27.6 \%)$ \\
\hline Nonsmoker & 189 & $(72.4 \%)$ \\
\hline \multicolumn{3}{|l|}{ Performance status } \\
\hline $\mathrm{KPS}<70$ & 74 & $(28.4 \%)$ \\
\hline $\mathrm{KPS} \geq 70$ & 187 & $(71.6 \%)$ \\
\hline Histology & $\mathrm{n}$ & $(\%)$ \\
\hline Adenocarcinoma & 240 & $(92.0 \%)$ \\
\hline Others & 21 & $(8.0 \%)$ \\
\hline \multicolumn{3}{|l|}{ Number of BM } \\
\hline$\leq 3$ & 175 & $(67.0 \%)$ \\
\hline$>3$ & 86 & $(33.0 \%)$ \\
\hline \multicolumn{3}{|l|}{ ECM } \\
\hline Yes & 117 & $(44.8 \%)$ \\
\hline No & 144 & $(55.2 \%)$ \\
\hline \multicolumn{3}{|l|}{ Symptomatic BM } \\
\hline With & 147 & $(56.3 \%)$ \\
\hline Without & 114 & $(43.7 \%)$ \\
\hline \multicolumn{3}{|l|}{ Synchronous BM } \\
\hline Yes & 189 & $(72.4 \%)$ \\
\hline No & 72 & $(27.6 \%)$ \\
\hline \multicolumn{3}{|l|}{ EGFR mutation } \\
\hline Exon 19 deletion & 138 & $(52.9 \%)$ \\
\hline L858R & 90 & $(34.5 \%)$ \\
\hline Others & 33 & $(12.6 \%)$ \\
\hline \multicolumn{3}{|c|}{ Intracranial local therapy } \\
\hline Yes & 195 & $(74.7 \%)$ \\
\hline No & 66 & $(25.3 \%)$ \\
\hline \multicolumn{3}{|l|}{ Chemotherapy } \\
\hline Without & 114 & $(43.7 \%)$ \\
\hline With & 147 & $(56.3 \%)$ \\
\hline \multicolumn{3}{|l|}{ DS-GPA score } \\
\hline $0-0.5$ & 42 & $(16.1 \%)$ \\
\hline $1-1.5$ & 107 & $(41.0 \%)$ \\
\hline $2-2.5$ & 86 & $(33.0 \%)$ \\
\hline$\geq 3$ & 26 & $(10.0 \%)$ \\
\hline \multicolumn{3}{|l|}{ Lung-molGPA score } \\
\hline $0-1$ & 7 & $(2.7 \%)$ \\
\hline $1.5-2$ & 71 & $(27.2 \%)$ \\
\hline $2.5-3$ & 125 & $(47.9 \%)$ \\
\hline$\geq 3.5$ & 58 & $(22.2 \%)$ \\
\hline
\end{tabular}

\section{Treatment}

Within the patients who received intracranial local therapy, 137 (70.2\%) received WBRT, 37 (19.0\%) received SRS or surgical resection, and $21(10.8 \%)$ had a combination of the above. There were 161 (61.7\%) patients who received EGFR TKIs as first line treatment. Among the 161 patients, 105 (65.2\%) were treated with icotinib, 38 (23.6\%) with gefitinib, and 18 $(11.2 \%)$ with erlotinib. There was no difference in OS between different EGFR TKIs ( $\mathrm{p}=0.087)$. Additionally, $117(72.7 \%)$ of these 161 patients received radiotherapy (RT), 86 received upfront $\mathrm{RT}$ (with an interval between RT and TKI treatment $\leq 1$ months), and 31 received deferral $R T$. We found no significant difference in OS between the two groups $(p=0.395)$.

\section{Postprogression Treatment}

It should be noted that osimertinib did not enter the Chinese market until April 2017; hence, only 24 patients received osimertinib after the progression of first-line EGFR TKIs. We found that patients treated with osimertinib achieved a survival benefit from its use $(\mathrm{p}=0.011)$.

\section{OS and Prognosis}

The median OS was 23.0 months $(95 \%$ CI 20.0-26.0, Fig. 2). By univariate analysis, significantly shorter OS was noted in patients older than 65 years $(p=0.025), K P S<70(p=0.003)$, and patients with ECM $(p=0.00)$. Patients who received chemotherapy were more likely to have longer OS than those who did not (28.0 vs 16.5, p=0.01). Meanwhile, patients who received local therapies also had longer OS than those who did not receive local therapies (24.9 vs 16.0 , $\mathrm{p}=0.00$ ). In addition, we found patients who revived SRS or surgical resection tended to have longer OS than those who underwent other local therapy methods (SRS or surgical resection 34.4 vs WBRT 23.2 vs combination 19.0), though it didn't reach the statistical significance $(p=0.067)$. There was also no difference in the patients respect to sex, smoking history, EGFR mutation type, number of BM. In multivariate analyses using multiple Cox proportional hazards models, we observed that performance status (KPS $<70, \mathrm{p}=0.010)$, extracranial metastases (ECM) at time of brain metastases $(p=0.001)$, receiving intracranial local treatment $(p=0.005)$ and chemotherapy $(p=0.005)$ were independent prognostic factors for OS, while age was not $(p=0.087)$. As for the prognostic index, survival by DS-GPA and Lung-molGPA group showed excellent separation between groups $(p=0.000 ; p=0.000$; Fig. 3 ) The survival times for all patients with DS-GPA scores of $0-0.5,1.0-1.5,2.0-2.5$, and more than 3 were 16.0, 17.7, 27.0 and 42.0 months, respectively. In addition, survival times for patients with Lung-molGPA scores of 0-1.0, 1.5-2.0, 2.5-3.0, and more than 3.5 were 15.0, 16.0, 23.7 and 32.0 months, respectively. Taken together, both the DS-GPA and Lung-molGPA models predicted the prognosis of NSCLC patients with $\mathrm{BM}(\mathrm{P}=0.000)$. However, the Lung-molGPA model did not show further superiority of its predictive effect. In multivariate analyses using multiple Cox proportional hazards models, we observed that performance status (KPS $\geq 70$; HR 0.639, 95\% CI 0.455-0.897, $\mathrm{p}=0.010$ ), ECM (HR 1.733, 95\% CI 1.256-2.391, $\mathrm{p}=0.001$ ), local therapy (HR 0.607, 95\% CI 0.430-0.857, $\mathrm{p}=0.005$ ) and chemotherapy (HR 0.629, 95\% CI 0.455-0.870, p=0.005) 
were independent prognostic factors for OS, while age was not (HR 1.436, 95\% CI 0.949-2.172, p=0.087;

Table 2, Fig. 4).

Table 2. Univariable and Multivariable Analyses of Covariables Associated With OS

\begin{tabular}{|c|c|c|c|c|c|c|}
\hline \multirow[b]{2}{*}{ Variables } & \multicolumn{3}{|c|}{ Univariate analysis } & \multicolumn{3}{|c|}{ Multivariate analysis-reduced } \\
\hline & Hazard ratio & $95 \% \mathrm{CI}$ & $p$ value & Hazard ratio & $95 \% \mathrm{CI}$ & $p$ value \\
\hline Age (>65 y/o) & 1.579 & $1.052-2.369$ & .025 & 1.436 & $0.949-2.172$ & .087 \\
\hline Gender (male) & 1.141 & $0.846-1.540$ & .384 & & & \\
\hline Smoking history (smoker) & 1.077 & $0.781-1.485$ & 649 & & & \\
\hline $\mathrm{KPS} \geq 70$ & 0.626 & $0.457-0.859$ & .003 & 0.639 & $0.455-0.897$ & .010 \\
\hline BM numbers $>3$ & 0.946 & $0.687-1.302$ & .729 & & & \\
\hline ECM & 1.744 & $1.287-2.362$ & .000 & 1.733 & $1.256-2.391$ & .001 \\
\hline EGFR mutation & 1.024 & $0.840-1.247$ & .343 & & & \\
\hline Intracranial local therapy & 0.546 & $0.392-0.761$ & .000 & 0.607 & $0.430-0.857$ & .005 \\
\hline Chemotherapy & 0.598 & $0.442-0.808$ & .001 & 0.629 & $0.455-0.870$ & .005 \\
\hline DS-GPA score & 0.675 & $0.558-0.818$ & .000 & & & \\
\hline Lung-molGPA score & 0.667 & $0.551-0.807$ & .000 & & & \\
\hline
\end{tabular}

Abbreviations: BM, brain metastases; DS-GPA, diagnosis-specific graded prognostic assessment; ECM, extracranial metastases; EGFR, epidermal growth factor receptor; KPS, Karnofsky performance score; OS, overall survival.

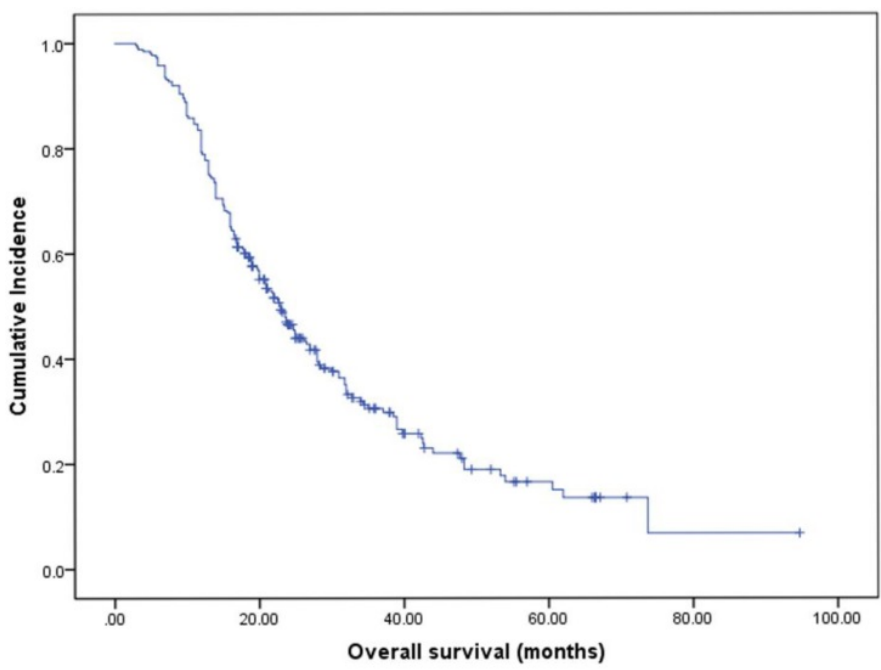

Figure 2. Kaplan-Meier curve illustrating OS of EGFR-mutant NSCLC patients with brain metastases showed a median OS (months from start of brain metastasis) of 23.0 months (95\% Cl: 20.0-26.0).
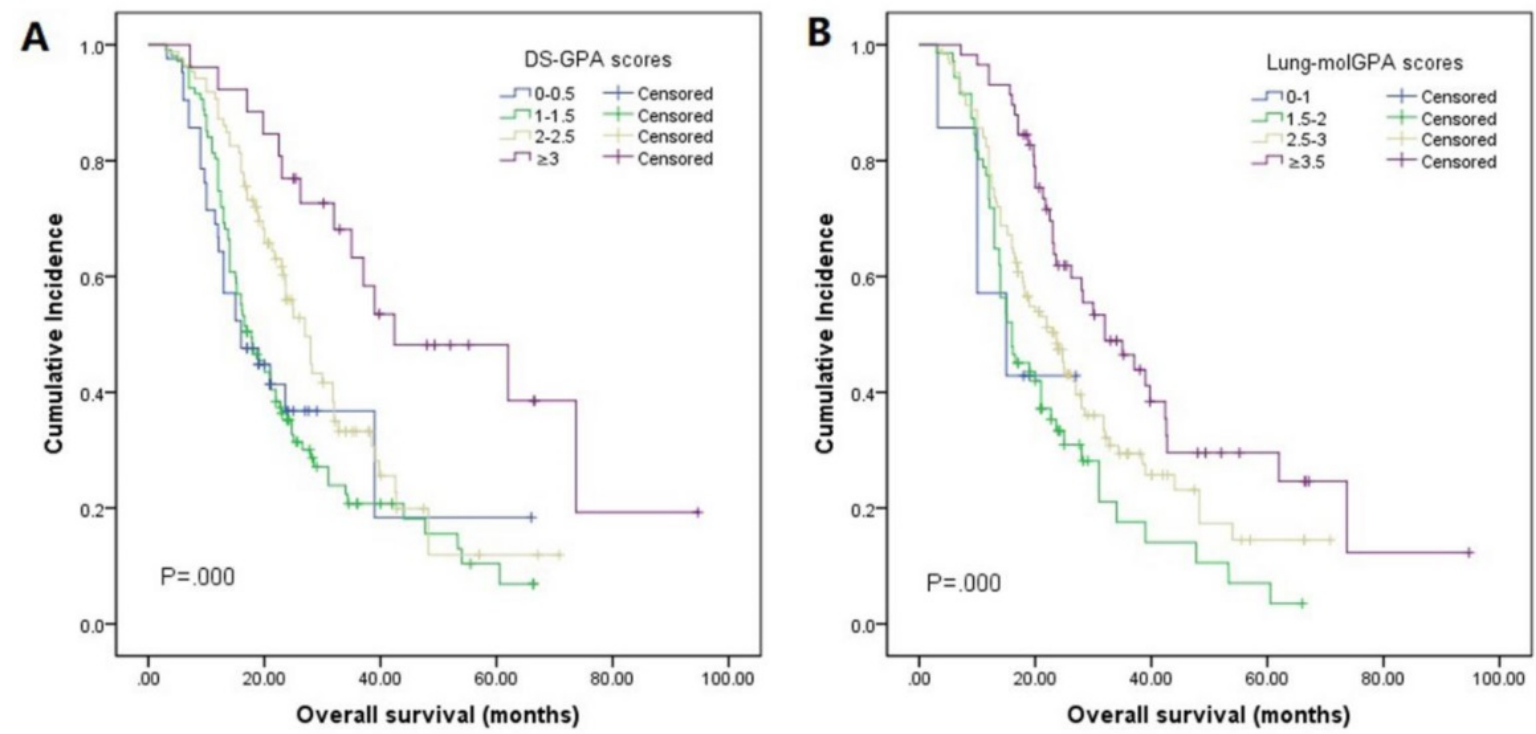

Figure 3. Kaplan-Meier analysis comparing overall survival in patients based on the DS-GPA and Lung-molGPA indexes. (A): For the DS-GPA, patients with a score of more than 3 had the longest median OS, 42.0 months ( $95 \% \mathrm{Cl}: 16.0-68.8$ ), followed by those with a score of $2-2.5$ (median OS=27.0 months; $95 \% \mathrm{Cl}: 22.6-31.4$ ), those with a score of $1-1.5$ (median OS=17.7 months; $95 \% \mathrm{Cl}$ : 14.4-21.0), and those with a score of 0-0.5 (median OS=16.0 months; $95 \%$ Cl: $8.6-23.4$ ). (B): For the Lung-molGPA, patients with a score of more than 3.5 had the longest median OS, 32.0 months ( $95 \% \mathrm{Cl}: 21.4-42.6$ ), followed by those with a score of $2.5-3$ (median OS=23.7 months; $95 \% \mathrm{Cl}$ : $18.9-28.5$ ), those with a score of 1.5-2 (median OS=16.0 months; $95 \% \mathrm{Cl}$ : 11.5-20.6), and those with a score of $0-1$ (median OS=15.0 months; $95 \% \mathrm{Cl}$ : $2.2-27.8$ ). 

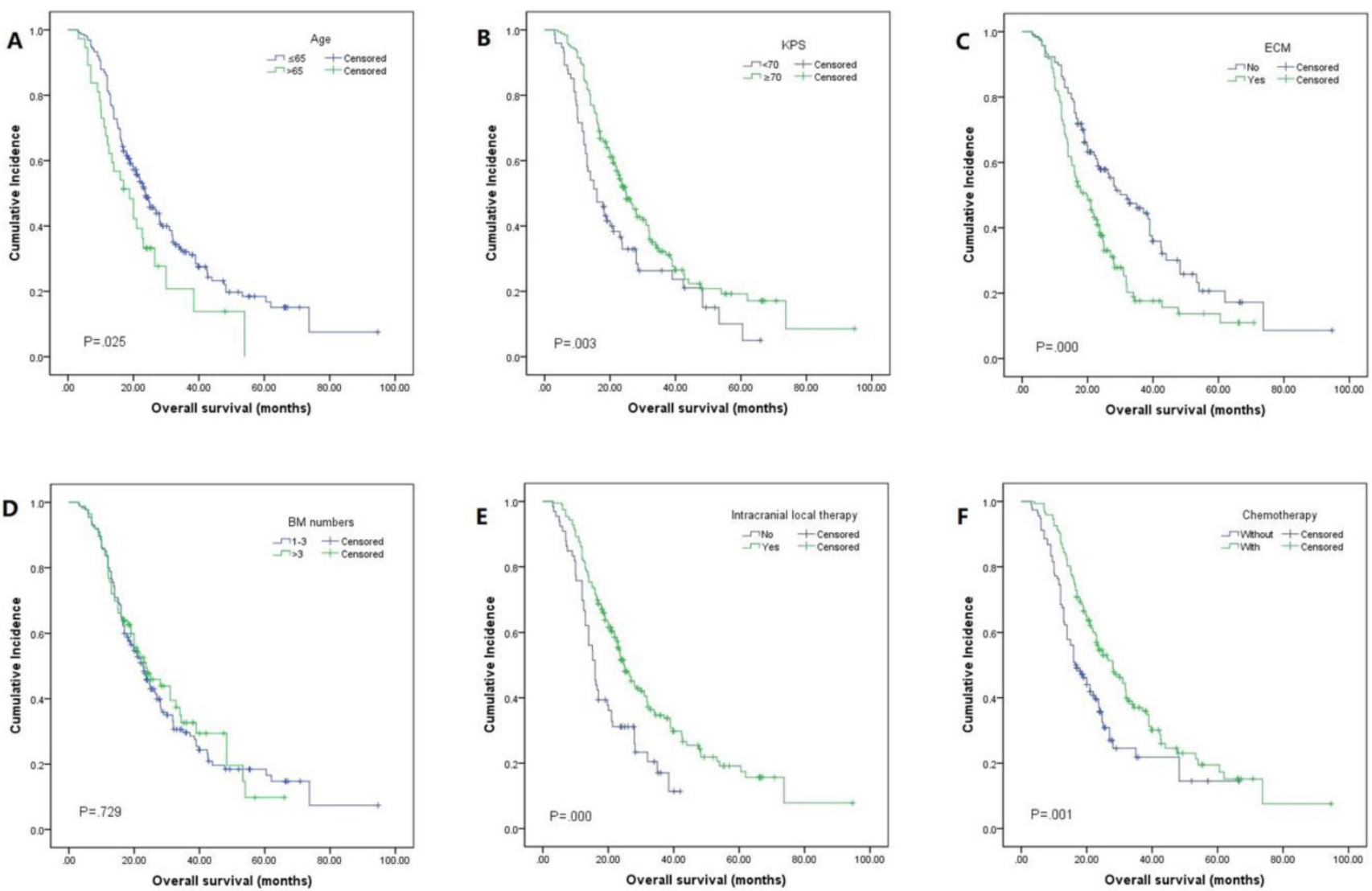

Figure 4. Kaplan-Meier analysis showing survival based on different factors for NSCLC: ( $A$ )age (mOS: 23.7 vs 18.8 months, $p=0.025)$ ), (B)KPS (mOS: 16.0 vs 24.9 months, $p=0.003$ ), (C)ECM (mOS: 32.0 vs 19.7 months, $p=0.000$ ), (D)BM numbers (mOS: 22.7 vs 23.6 months, $p=0.729$ ), (E)intracranial local therapy (mOS: 16.0 vs 24.9 months, $\mathrm{p}=0.000$ ), (F)chemotherapy (mOS: 16.5 vs 28.0 months, $\mathrm{p}=0.001$ )

\section{Discussion}

In this study, we found that KPS, absence of $\mathrm{ECM}$, receiving intracranial local therapy and the administration of chemotherapy were independent prognostic factors for OS in patients harboring an EGFR mutation and brain metastases, in real-world practice. While age, number of BM and EGFR mutation type showed no impact on survival. We also found that the sequence of RT and EGFR TKIs had no significant effect on prognosis. The DS-GPA and Lung-molGPA indexes were still valid in predicting the prognosis of EGFR-mutant patients with BM, but the Lung-molGPA index did not show further superiority in NSCLC patients with BM and clear gene status.

Extracranial metastases as a prognostic predictor have been reviewed previously. Regardless of the Recursive Partitioning Analysis (RPA) or the DS-GPA index or any other prognostic index, ECM has always been an independent prognosticator influencing survival and is most often the strongest one[14-17]. In our study, ECM was once again confirmed to be prognostic. It is reasonable to assume that patients with ECM would live shorter periods than those without ECM. The underlying mechanism could be that patients with ECM once had a heavy disease burden. Treatments for these patients should be both intracranial and extracranial, which might be the possible reason for their worse prognosis. As Kocher et al. pointed out, the presence of ECM may also influence survival by reseeding the brain parenchyma, thus forming another potential source of new brain metastasis, as indicated by the presence of multiple extracranial metastases[18]. Meanwhile, patients with good control of ECM-associated activities have a certain curative rate and can benefit more from local treatment.

The number of $\mathrm{BM}$ has been reported to be a poor prognostic factor in NSCLC. Our study, however, found that the number of BM was not associated with survival. This finding may imply that the number of $\mathrm{BM}$ is not a reliable prognostic factor in the setting of EGFR mutations and TKI therapy. Previous database analysis of the prognostic indexes included EGFR wild-type group, and this inclusion may have affected the accuracy of prognosis for EGFR-mutant patients $[3,15,17]$.We assume that TKIs have a good control of intracranial lesions in patients with EGFR mutations and BM. Therefore, the number of BM may not influence the efficacy of TKIs. 
In our analysis of different EGFR mutation subgroups, we found EGFR mutation type had no impact on OS. Median OS of patients with exon 19 deletion, L858R mutation and other EGFR mutations in our study was 23.6 months, 19.7 months and 27.0 months, respectively $(p=0.343$ ). Former studies have found exon 19 deletion achieved better OS than L858R mutation in NSCLC patients receiving EGFR-TKI treatments $[4,19,20]$. A similar result was also found in EGFR-mutant patients with BM [6]. Nevertheless, there were reports that indicated that the type of EGFR mutation was not predictive for the development of brain metastases or the survival of patients [21]. A number of studies have reported that the efficacies of EGFR-TKIs are distinct among different mutant subtypes. However, most patients with BM would be willing to undergo intensification therapy for intracranial lesions. This result could be the reason that, in our study, EGFR mutation type had no apparent impact on the survival of patients with BM.

A series of studies evaluating the use of cranial local therapies in patients with $\mathrm{BM}$ has been performed and suggests a survival benefit from local therapy. In our study, we noted that the administration of local therapy can significantly improve survival and was a prognostic predictor. In the current era of targeted therapy, EGFR TKIs alone have demonstrated activity against intracranial disease in EGFR-mutant NSCLC [11, 22, 23]. In addition, a systematic review and meta-analysis of 12 studies found that, relative to treatment with TKIs alone, cranial radiotherapy followed by EGFR TKIs improves intracranial control and survival outcomes in EGFR-mutant NSCLC with BM [24]. Multiple studies have shown patients with EGFR mutation to be highly radiosensitive in both the preclinical and clinical settings, thereby making the ablation of $\mathrm{BM}$ a distinct possibility $[25,26]$. It was previously reported that local control rates of SRS in the treatment of EGFR-mutant brain metastases had reached $100 \%$ and $93 \%[25,27]$. Another multi-institutional analysis also revealed the impact of RT followed by EGFR TKIs on patient outcomes was most pronounced in patients with a more favorable prognosis (dsGPA,2-4)[5]. For patients with good DS-GPA scores, more active and reasonable intracranial local treatment is recommended.

In the subgroup of patients who received EGFR TKIs as first-line treatment, no significant difference in OS was noted between patients who received upfront RT and those who did not ( $p=0.395)$, which is a different result than reported in previous studies. A retrospective study found that the use of upfront EGFR-TKI, and deferral of RT, would result in inferior survival in patients with EGFR-mutant NSCLC who develop BM [28]. Extensive efforts have focused on the optimal sequence of RT for BM patients. Magnuson et al. also noted significantly worse survival in those who had delayed brain RT, regardless of whether it was SRS or WBRT [5, 29]. However, this finding needs to be interpreted with caution because the relatively small sample size and retrospective design may lead to selection bias for treatment. Prospective, multi-institution randomized trials are urgently needed to determine the optimal treatment combination or sequence. The BRAIN study indicated that, in patients with EGFR-mutant NSCLC and multiple brain metastases, icotinib was more recommended as a first-line therapeutic option over WBI treatment [12]. It is recommended that through the use of highly active targeted therapies, WBRT can be safely postponed in patients with EGFR mutation and BM [30]. However, the optimal sequences between EGFR TKI and RT are still controversial.

We also found that the administration of chemotherapy on the basis of EGFR TKIs can significantly improve the survival and was a prognostic predictor. Accordingly, chemotherapy could achieve better systemic disease control. In our study, 147 patients received chemotherapy, with 97 $(66.0 \%)$ receiving pemetrexed-based chemotherapy. It is reported that some drugs might, to some extent, penetrate the blood-brain barrier (BBB) and gain intracranial control. Barlesi et al. previously demonstrated that pemetrexed-based chemotherapy had the greatest activity, as well as a favorable safety profile, in managing NSCLC patients with inoperable BM [31]. It should be noted that this study did not observe whether the adoption of chemotherapy on the basis of EGFR TKIs would bring more adverse effect.

The results of our study confirmed the DS-GPA index remains a robust tool and was useful for estimating survival in EGFR-mutant patients with BM. However, the Lung-molGPA index updated by Sperduto et al did not appear superior when compared to DS-GPA in the gene-specific NSCLC patients with BM in this study. Perhaps a more accurate and user-friendly tool could be developed for patients with specific oncogenic driver genes.

This study is limited by its retrospective nature. Because it is a single-institutional study, there may have been bias in choosing patients for enrollment. Therefore, the results reported here are not entirely representative of a large sample population. Another limitation of this study is that follow-up data on toxicities, cognitive impairment and quality of life were lacking; therefore, we were unable to analyze these factors. 
In conclusion, this study provided real-world evidence for the survival and prognosis of EGFR-mutant NSCLC patents with BM. Performance status (KPS $\geq 70$ ), absence of ECM, the administration of local treatment or chemotherapy might all predict superior OS in EGFR-mutant NSCLC patients who develop brain metastases. While the DS-GPA and Lung-molGPA indexes were still robust tools, we propose a more accurate and user-friendly tool that applies to patients including specific gene status and BM. Further studies with large sample sizes are warranted.

\section{Competing Interests}

The authors have declared that no competing interest exists.

\section{References}

1. Dempke WC, Edvardsen K, Lu S, Reinmuth N, Reck M, Inoue A. Brain Metastases in NSCLC - are TKIs Changing the Treatment Strategy? Anticancer Research. 2015; 35: 5797.

2. Gavrilovic IT, Posner JB. Brain metastases: epidemiology and pathophysiology. Journal of Neuro-Oncology. 2005; 75: 5-14.

3. Sperduto PW, Yang TJ, Beal K, Pan H, Brown PD, Bangdiwala A, et al. Estimating Survival in Patients With Lung Cancer and Brain Metastases: An Update of the Graded Prognostic Assessment for Lung Cancer Using Molecular Markers (Lung-molGPA). Jama Oncol. 2017; 3: 827.

4. H S, L L, T T, M N, M S, II W, et al. Clinical and biological features associated with epidermal growth factor receptor gene mutations in lung cancers. J Natl Cancer Inst. 2005; 98: 362-3.

5. Magnuson WJ, Lester-Coll NH, Wu AJ, Yang TJ, Lockney NA, Gerber NK, et al. Management of Brain Metastases in Tyrosine Kinase Inhibitor-Naïve Epidermal Growth Factor Receptor-Mutant Non-Small-Cell Lung Cancer: A Retrospective Multi-Institutional Analysis. Journal of Clinical Oncology Official Journal of the American Society of Clinical Oncology. 2017; 35: 1070.

6. Fan Y, Xu Y, Gong L, Fang L, Lu H, Qin J, et al. Effects of icotinib with and without radiation therapy on patients with EGFR mutant non-small cell lung cancer and brain metastases. Scientific Reports. 2017; 7: 45193.

7. Preusser M, Winkler F, Valiente M, Manegold C, Moyal E, Widhalm G, et al. Recent advances in the biology and treatment of brain metastases of non-small cell lung cancer: summary of a multidisciplinary roundtable discussion. Esmo Open. 2018; 3: e000262.

8. Zhao J, Chen M, Zhong W, Zhang L, Li L, Xiao Y, et al. Cerebrospinal fluid concentrations of gefitinib in patients with lung adenocarcinoma. Clinical Lung Cancer. 2013; 14: 188-93.

9. Porta R, Sánchez-Torres JM, Paz-Ares L, Massutí B, Reguart N, Mayo C, et al. Brain metastases from lung cancer responding to erlotinib: the importance of EGFR mutation. European Respiratory Journal. 2011; 37: 624.

10. Fan Y, Huang Z, Fang L, Miao L, Gong L, Yu H, et al. A phase II study of icotinib and whole-brain radiotherapy in Chinese patients with brain metastases from non-small cell lung cancer. Cancer Chemotherapy \& Pharmacology 2015; 76: 517

11. Iuchi T, Shingyoji M, Sakaida T, Hatano K, Nagano O, Itakura M, et al. Phase II trial of gefitinib alone without radiation therapy for Japanese patients with brain metastases from EGFR-mutant lung adenocarcinoma. Lung Cancer. 2013; 82: 282-7.

12. Yang JJ, Zhou C, Huang Y, Feng J, Lu S, Song Y, et al. Icotinib versus whole-brain irradiation in patients with EGFR-mutant non-small-cell lung cancer and multiple brain metastases (BRAIN): a multicentre, phase 3, open-label, parallel, randomised controlled trial. Lancet Respir Med. 2017; 5: 707.

13. $\mathrm{HW}, \mathrm{M} \mathrm{O}, \mathrm{YK}, \mathrm{M} \mathrm{S}, \mathrm{YS}, \mathrm{YY}$, et al. [New response evaluation criteria in solid tumours-revised RECIST guideline (version 1.1)]. Gan to Kagaku Ryoho Cancer \& Chemotherapy. 2009; 36: 2495-501

14. Jeremic B, Becker G, Plasswilm L, Bamberg M. Activity of extracranial metastases as a prognostic factor influencing survival after radiosurgery of brain metastases. Journal of Cancer Research \& Clinical Oncology. 2000; 126: 475-80.

15. Sperduto PW, Kased N, Roberge D, Xu Z, Shanley R, Luo X, et al. Summary report on the graded prognostic assessment: an accurate and facile diagnosis-specific tool to estimate survival for patients with brain metastases. Journal of clinical oncology : official journal of the American Society of Clinical Oncology. 2012; 30: 419-25.

16. Sperduto PW, Berkey B, Gaspar LE, Mehta M, Curran W. A new prognostic index and comparison to three other indices for patients with brain metastases: an analysis of 1,960 patients in the RTOG database. International Journal of Radiation Oncology Biology Physics. 2008; 70: 510-4.

17. Gaspar L, Scott C, Rotman M, Asbell S, Phillips T, Wasserman T, et al. Recursive partitioning analysis (RPA) of prognostic factors in three Radiation Therapy Oncology Group (RTOG) brain metastases trials. International journal of radiation oncology, biology, physics. 1997; 37: 745-51.

18. Kocher M, Voges J, Müller RP, Sturm V, Müller J, Staar S, et al. LINAC Radiosurgery for Patients with a Limited Number of Brain Metastases. Journal of Radiosurgery. 1998; 1: 9-15.

19. GJ R, W P, D P, AR L, N R, ES V, et al. Clinical course of patients with non-small cell lung cancer and epidermal growth factor receptor exon 19 and exon 21 mutations treated with gefitinib or erlotinib. Clinical Cancer Research. 2006; 12: 839-44.

20. Yang JC, Wu YL, Schuler M, Sebastian M, Popat S, Yamamoto N, et al. Afatinib versus cisplatin-based chemotherapy for EGFR mutation-positive lung adenocarcinoma (LUX-Lung 3 and LUX-Lung 6): analysis of overall survival data from two randomised, phase 3 trials. Lancet Oncology. 2015; 16: 141-51.

21. Stanic K, Hitij NT. P16. Is type of EGFR mutation predictive for incidence and survival in lung adenocarcinoma patients with brain metastases? 2014; 3 .

22. Park SJ, Kim HT, Lee DH, Kim KP, Kim SW, Suh C, et al. Efficacy of epidermal growth factor receptor tyrosine kinase inhibitors for brain metastasis in non-small cell lung cancer patients harboring either exon 19 or 21 mutation. Lung Cancer. 2012; 77: 556-60.

23. Wu YL, Zhou C, Cheng Y, Lu S, Chen GY, Huang C, et al. Erlotinib as second-line treatment in patients with advanced non-small-cell lung cancer and asymptomatic brain metastases: a phase II study (CTONG-0803). Annals of Oncology. 2013; 24: 993-9.

24. Soon YY, Leong CN, Koh WY, Tham IW. EGFR tyrosine kinase inhibitors versus cranial radiation therapy for EGFR mutant non-small cell lung cancer with brain metastases: a systematic review and meta-analysis. Radiotherapy and oncology : journal of the European Society for Therapeutic Radiology and Oncology. 2015; 114: 167-72.

25. Johung KL, Yao X, Li F, Yu JB, Gettinger SN, Goldberg S, et al. A Clinical Model for Identifying Radiosensitive Tumor Genotypes in Non-Small Cell Lung Cancer. Clinical Cancer Research An Official Journal of the American Association for Cancer Research. 2013; 19: 5523-32.

26. Spano JP, Fagard R, Soria JC, Rixe O, Khayat D, Milano G. Epidermal growth factor receptor signaling in colorectal cancer: preclinical data and therapeutic perspectives. Annals of Oncology. 2005; 16: 189-94.

27. Wang TJ, Saad S, Qureshi YH, Jani A, Nanda T, Yaeh AM, et al. Does lung cancer mutation status and targeted therapy predict for outcomes and local control in the setting of brain metastases treated with radiation? Neuro-oncology. 2015; 17: 1022-8.

28. Magnuson WJ, Yeung JT, Guillod PD, Gettinger SN, Yu JB, Chiang VL. Impact of Deferring Radiation Therapy in Patients With Epidermal Growth Factor Receptor-Mutant Non-Small Cell Lung Cancer Who Develop Brain Metastases. International journal of radiation oncology, biology, physics. 2016; 95: 673-9.

29. Zhou L, Deng L, Lu Y. Epidermal Growth Factor Receptor Mutations in Non-Small-Cell Lung Cancer With Brain Metastasis: Can Up-Front Radiation Therapy Be Deferred or Withheld? Journal of Clinical Oncology. 2017; 35: 1033-5.

30. Martinez P, Mak RH, Oxnard GR. Targeted Therapy as an Alternative to Whole-Brain Radiotherapy in EGFR-Mutant or ALK-Positive Non-Small-Cell Lung Cancer With Brain Metastases. JAMA Oncol. 2017; 3: 1274-5.

31. Barlesi F, Gervais R, Lena H, Hureaux J, Berard H, Paillotin D, et al. Pemetrexed and cisplatin as first-line chemotherapy for advanced non-small-cell lung cancer (NSCLC) with asymptomatic inoperable brain metastases: a multicenter phase II trial (GFPC 07-01). Annals of Oncology. 2011; 22: 2466-70. 